\section{Somatostatin Receptor-Targeted Radiopeptide Therapy in Patients with Progressive Unresectable Meningioma}

TO THE EDITOR: Marincek et al. (1) recently presented a study aimed at evaluating the long-term outcome after treatment with ${ }^{90}$ Y-DOTATOC and ${ }^{177} \mathrm{Lu}$-DOTATOC in patients affected by unresectable progressive meningioma. In that study, 34 patients from Europe and North America were treated with ${ }^{90}$ Y-DOTATOC and ${ }^{177} \mathrm{Lu}$-DOTATOC until tumor progression or permanent toxicity occurred. The authors concluded that ${ }^{90}$ Y-DOTATOC and ${ }^{177} \mathrm{Lu}$-DOTATOC are promising tools for the treatment of progressive unresectable meningioma, especially in patients with high radiopharmaceutical accumulation in the tumor.

Recently, my group reported on peptide receptor radionuclide therapy in 8 patients who had been referred to the unit of nuclear medicine of our university hospital with a diagnosis of meningioma (6 patients) or meningiomatosis (2 patients) (2). On ${ }^{111}$ In-pentetreotide scintigraphy, all patients showed grade 2 or 3 tumoral uptake; moreover, the median tumor-to-nontumor ratio as evaluated by a standard region-of-interest method on the 24-h scan was 5.7, ranging from 2.9 to 9.1. All patients had been treated with ${ }^{111}$ In-pentetreotide at high therapeutic activities (2-4 cycles, median activity per cycle, $7 \mathrm{GBq}$; cumulative activity range, 4.8-29 GBq); one patient had been previously treated with 6 cycles of ${ }^{90}$ Y-DOTATOC (cumulative activity, $13.3 \mathrm{GBq}$; the patient had a mild impairment of renal function); in 2 patients, a cocktail of ${ }^{111} \mathrm{In}$ pentetreotide and $\beta$-emitting radiolabeled peptides $\left({ }^{90} \mathrm{Y}\right.$-DOTATOC and ${ }^{177} \mathrm{Lu}$-DOTATATE) had been administered. Disease response was evaluated on the basis of the Southwest Oncology Group criteria: partial response was noted in 2 patients, stable disease in 5 patients, and disease progression in 1 patient. No acute toxicity or neurologic or renal function impairment occurred. Mild and transient hematologic toxicity was found in 4 patients. No significant correlations were noted between objective response and patient age, World Health Organization tumor grade, baseline Karnofsky performance score, baseline disease state, or cumulative dose.

The results of our study are similar to and support the results of Marincek et al. for peptide receptor radionuclide therapy in patients with progressive unresectable meningiomas, even after multimodal treatment, especially in patients with limited treatment options or recurrent lesions. Moreover, our study found that treatment with ${ }^{111}$ In-pentetreotide was well tolerated and effective in patients with meningiomas or meningiomatosis. We thus concluded that, considering the lack of significant toxicity, peptide receptor radionuclide therapy of meningiomas using ${ }^{111}$ In-pentetreotide can be proposed even today if the use of ${ }^{177} \mathrm{Lu}$ - or ${ }^{90}$ Y-DOTA-peptides seems unsafe, namely in patients with renal impairment or toxicity. I further suggest the use of ${ }^{111} \mathrm{In}$-pentetreotide or ${ }^{177} \mathrm{Lu}$-DOTA-peptides (characterized by a shorter penetration range than ${ }^{90}$ Y-DOTA-peptides) in patients who have progressive unresectable meningiomas involving

COPYRIGHT (c) 2016 by the Society of Nuclear Medicine and Molecular Imaging, Inc. critical structures (vascular and neural ones) to avoid eventual complications from radiation-induced edema.

\section{REFERENCES}

1. Marincek N, Radojewski P, Dumont RA, et al. Somatostatin receptor-targeted radiopeptide therapy with ${ }^{90} \mathrm{Y}$-DOTATOC and ${ }^{177} \mathrm{Lu}$-DOTATOC in progressive meningioma: long-term results of a phase II clinical trial. J Nucl Med. 2015;56:171-176.

2. Minutoli F, Amato E, Sindoni A, et al. Peptide receptor radionuclide therapy in patients with inoperable meningiomas: our experience and review of the literature. Cancer Biother Radiopharm. 2014;29:193-199.

\section{Alessandro Sindoni \\ University of Messina Via Consolare Valeria 198125 Messina, Italy \\ E-mail: alessandrosindoni@alice.it}

Published online Feb. 25, 2016.

DOI: 10.2967/jnumed.116.172312

REPLY: We appreciate the thoughtful comments regarding our paper "Somatostatin receptor-targeted radiopeptide therapy with ${ }^{90}$ Y-DOTATOC and ${ }^{177} \mathrm{Lu}$-DOTATOC in progressive meningioma: long-term results of a phase II clinical trial" (1), and we are grateful for the opportunity to be part of the ongoing discussion concerning the management of progressive meningiomas.

Meningiomas are the most common primary brain tumors, with an incidence 20 times higher than that of neuroendocrine tumors. About $80 \%$ of meningiomas are benign and curable with gross total resection, whereas about $20 \%$ of meningiomas are atypical or anaplastic and demonstrate malignant potential, significantly higher recurrence rates, and shorter survival. There is currently no standard of care for patients with progressive meningiomas, and the National Comprehensive Cancer Network recommends hydroxyurea, $\alpha$-interferon, and somatostatin analogs (2).

Our study as well as other recent work $(3,4)$ suggests that somatostatin receptor-targeted radiopeptides represent a promising therapeutic option for patients with progressive meningiomas. Herein lies an opportunity for the field of nuclear medicine, in collaboration with neurooncology, radiation oncology, and neurosurgery, to translate this tool from a promising option into a validated therapy.

There may be some clinical utility in determining the ideal radiopeptide and radioisotope combination, such as DOTATOC versus DOTATATE or ${ }^{90} \mathrm{Y}$ versus ${ }^{177} \mathrm{Lu}$. However, establishing the value of radiopeptide therapy in progressive meningioma, as a single treatment or in combination with other drugs, will ultimately yield true clinical benefit. With this in mind, we look forward to an interdisciplinary preclinical and clinical approach.

Prospective clinical trials will be essential to compare the benefits and harms of somatostatin receptor-targeted radiopeptides with those of the currently recommended therapeutics hydroxyurea, $\alpha$-interferon, and somatostatin analogs in patients with progressive meningiomas. These studies are particularly promising given the high expression of somatostatin receptors in meningiomas (5). In addition, metaanalyses evaluating existing data regarding these treatments may prove helpful. 